\title{
Costs and consequences of treatment for mild gestational diabetes mellitus - evaluation from the ACHOIS randomised trial John R Moss ${ }^{1}$, Caroline A Crowther*2, Janet E Hiller ${ }^{1}$, Kristyn J Willson ${ }^{1}$, Jeffrey S Robinson ${ }^{2}$ for The Australian Carbohydrate Intolerance Study in Pregnant Women (ACHOIS) Trial Group
}

\author{
Address: ${ }^{1}$ Discipline of Public Health, The University of Adelaide, Adelaide, South Australia, 5005, Australia and ${ }^{2}$ Discipline of Obstetrics and \\ Gynaecology, The University of Adelaide, Women's and Children's Hospital, King William Road, North Adelaide, South Australia, 5006, Australia \\ Email: John R Moss - john.moss@adelaide.edu.au; Caroline A Crowther* - caroline.crowther@adelaide.edu.au; \\ Janet E Hiller - janet.hiller@adelaide.edu.au; Kristyn J Willson - kristyn.willson@adelaide.edu.au; \\ Jeffrey S Robinson - jeffrey.robinson@adelaide.edu.au; The Australian Carbohydrate Intolerance Study in Pregnant Women (ACHOIS) Trial \\ Group - caroline.crowther@adelaide.edu.au \\ * Corresponding author
}

Published: 28 October 2007

BMC Pregnancy and Childbirth 2007, 7:27 doi:10.1 I86/I47|-2393-7-27

This article is available from: http://www.biomedcentral.com/147I-2393/7/27

(c) 2007 Moss et al; licensee BioMed Central Ltd.

This is an Open Access article distributed under the terms of the Creative Commons Attribution License (http://creativecommons.org/licenses/by/2.0), which permits unrestricted use, distribution, and reproduction in any medium, provided the original work is properly cited.

\begin{abstract}
Background: Recommended best practice is that economic evaluation of health care interventions should be integral with randomised clinical trials. We performed a cost-consequence analysis of treating women with mild gestational diabetes mellitus by dietary advice, blood glucose monitoring and insulin therapy as needed compared with routine pregnancy care, using patientlevel data from a multi-centre randomised clinical trial.

Methods: Women with a singleton pregnancy who had mild gestational diabetes diagnosed by an oral glucose-tolerance test between 24 and 34 weeks' gestation and their infants were included. Clinical outcomes and outpatient costs derived from all women and infants in the trial. Inpatient costs derived from women and infants attending the hospital contributing the largest number of enrolments (26.1\%), and charges to women and their families derived from a subsample of participants from that hospital (in 2002 Australian dollars). Occasions of service and health outcomes were adjusted for maternal age, ethnicity and parity. Analysis of variance was used with bootstrapping to confirm results. Primary clinical outcomes were serious perinatal complications; admission to neonatal nursery; jaundice requiring phototherapy; induction of labour and caesarean delivery. Economic outcome measures were outpatient and inpatient costs, and charges to women and their families.
\end{abstract}

Results: For every 100 women with a singleton pregnancy and positive oral glucose tolerance test who were offered treatment for mild gestational diabetes mellitus in addition to routine obstetric care, $\$ 53,985$ additional direct costs were incurred at the obstetric hospital, $\$ 6,52 \mathrm{I}$ additional charges were incurred by women and their families, 9.7 additional women experienced induction of labour, and 8.6 more babies were admitted to a neonatal nursery. However, 2.2 fewer babies experienced serious perinatal complication and 1.0 fewer babies experienced perinatal death. The incremental cost per additional serious perinatal complication prevented was $\$ 27,503$, per perinatal death prevented was $\$ 60,506$ and per discounted life-year gained was $\$ 2,988$.

Conclusion: It is likely that the general public in high-income countries such as Australia would find reductions in perinatal mortality and in serious perinatal complications sufficient to justify additional health service and personal monetary charges. Over the whole lifespan, the incremental cost per extra life-year gained is highly favourable.

Trial Registration: Australian Clinical Trials Registry ACTRNI 2606000294550 


\section{Background}

Since resources are inescapably scarce, health care interventions should be assessed for their impact on costs as well as on clinical outcomes. Recommended best practice is that economic evaluation should be integral with randomised clinical trials [1]. This has the advantage of generating patient-level data for a relatively modest research protocol cost.

The background, methods and pregnancy outcomes of the Australian Carbohydrate Intolerance Study in Pregnant Women (ACHOIS) trial have been reported elsewhere [2]. Briefly, a multi-centre randomised clinical trial was conducted from 1993 to 2003 to determine whether treatment of women with mild gestational diabetes mellitus reduced the risk of serious perinatal complications. Women between 24 and 34 weeks' gestation who had mild gestational diabetes were randomly assigned to receive dietary advice, blood glucose monitoring, and insulin therapy as needed (Intervention Group) or routine care according to standard practice at each centre. (Routine-Care Group).

Eligible women had a singleton or twin pregnancy between 16 and 30 weeks' gestation, attended antenatal clinics at the collaborating hospitals, and after screening positive for risk of gestational diabetes had a $75 \mathrm{~g}$ oral glucose-tolerance (OGTT) test at 24 to 34 weeks' gestation in which the venous plasma glucose level was less than 7.8 $\mathrm{mmol} / \mathrm{L}$ after an overnight fast and 7.8 to $11.0 \mathrm{mmol} / \mathrm{L}$ at two hours [3]. Women with more severe glucose impairment were not eligible for the ACHOIS trial.

\section{Methods}

The economic evaluation took the perspective of the health system and its patients and compared the direct costs of the additional resources used from randomisation until the time of postnatal hospital discharge of the woman or her baby, whichever occurred last, with the increments in health outcome for both the woman and the baby in a cost-consequences analysis $[4,5]$. Women with a twin pregnancy were excluded from this economic evaluation because it was anticipated that their likely increased use of resources could not be robustly estimated due to their expected small numbers in the trial. This analysis included all costs, but only those primary clinical outcomes (consequences) that achieved a P-value less than 0.05 in the main trial after adjustment for the confounders of maternal age, race or ethnic group, and parity. The economic evaluation was designed to take the form of a cost-consequences analysis, since trading-off between the utilities (preference values) of mother and infant would entail difficult conceptual and ethical issues. The intention was to provide relevant information about the incre- mental value of the intervention to assist decision-makers in setting priorities for health resource allocation.

The costing reported in this paper began at the moment of randomisation, and ended at the initial postnatal hospital discharge of the woman or her baby, whichever occurred last. Direct costs were measured to the health system and charges to the woman and her family. Cost was calculated as the number of occasions of each service multiplied by its unit cost. More than $98 \%$ of participants were public hospital patients.

Obstetric hospital outpatient occasions of service after enrolment were collected from the trial data for all women with a singleton pregnancy. Unit costs were obtained from sources congruent with the Manual of Resource Items for use in Major Submissions to the Australian Pharmaceutical Benefits Advisory Committee involving Economic Analyses [6]. The Manual recognises sources that include the Schedule of Medicare Benefits [7], the Pharmaceutical Benefits Schedule [8], and the Department of Veterans' Affairs Schedule of Fees [9]. Unit costs of relevant services are presented in Table 1.

Inpatient costing was only able to be performed for women and babies who were born at the Women's and Children's Hospital (WCH), Adelaide, Australia; the hospital which recruited the largest number of women (261/ 1,$000 ; 26.1 \%$ ) and which is typical of a large metropolitan tertiary care centre in Australia. The participating clinicians in the ACHOIS multi-centre trial had agreed to a common clinical management protocol. The trial began before computerised inpatient cost information systems became routinely available in the participating hospitals. From 1995-96 onwards, inpatient costs were obtained from Trendstar ${ }^{\circledR}$ (McDonnell Douglas Information Systems), the WCH patient costing system, based on input from several feeder systems, including nursing dependency levels. In this system, inpatient separations are case mix-classified according to Australian-Refined Diagnosis Related Groups. Cost weights come from the National Hospital Cost Data Collection [10] and include overheads. Inpatient costs for the baby were added to those of the mother.

Charges to women and their families were obtained by a questionnaire survey of a sample of 108 South Australian study participants (majority from the $\mathrm{WCH}$ ) after funding for this became available, from January 1997 to June 2003. The themes covered in the questionnaire included time off work and costs for food, hospital parking, childcare, and blood sugar monitoring equipment. Data from general practitioners or community service providers were not collected because of research budgetary constraints. Costs were expressed in calendar year 2002 Australian 
Table I: Unit costs of health services associated with the ambulatory management of gestational diabetes mellitus.

\begin{tabular}{lll}
\hline Item & Unit cost (Australian dollars 2002) & \multicolumn{1}{c}{ Source } \\
\hline $\begin{array}{l}\text { Antenatal clinic visits } \\
\text { Physician clinic visits: } \\
\text { initial }\end{array}$ & $\$ 28.75$ & MBS item 16500 \\
$\quad$ subsequent & $\$ 119.35$ & MBS items II0, II6 (consultant physician) \\
$\begin{array}{l}\text { Dietician visits: } \\
\text { initial }\end{array} \quad \$ 59.75$ & DVA \\
$\quad$ subsequent & $\$ 63.84$ & DT-03 $(\$ 5.32 * 12)$ \\
Diabetes educator & $\$ 31.92$ & DT-22 $(\$ 5.32 * 6)$ \\
Insulin therapy & $\$ 31.92$ & As for dietician subsequent visit (see above) \\
& $\$ 46.26$ per week & Weighted average DPMQ for II PBS insulin \\
& & items; Mean duration 6.4 weeks Intervention \\
& & Group, 5.4 weeks Routine-Care Group
\end{tabular}

MBS = Medicare Benefits Schedule I November 200I including I May 2002 Supplement; DVA = Department of Veterans' Affairs schedule of fees for services in a public hospital, 2002; PBS = Schedule of Pharmaceutical Benefits, November 200I; weighted by Health Insurance Commission data on overall population use; DPMQ = Dispensed Price for Maximum Quantity.

dollars, adjusted by the Consumer Price Index (CPI) [11] Based on 2002 purchasing power parities, one Australian dollar would convert to 0.74 US dollars, 0.48 UK pounds or 0.66 Euros [12].

The statistical analysis of the main ACHOIS trial with a sample size of 1000 women has been described elsewhere [2]. A pragmatic judgment was made that the sample size for the economic evaluation should be determined by the limits of detection of differences in the primary outcome measures in the main trial. The power of the study to show differences in total health service costs was $7.5 \%$ and for patient changes $17.5 \%$, assuming normality. As is common in health economic evaluation, the distribution of the costs per patient of managing gestational diabetes mellitus was skewed; hence bootstrapping (using 10000 resamples) was used to confirm the results of the analysis of variance [13]. All occasions of service and health outcomes were adjusted by maternal age, race or ethnic group, and parity.

\section{Results}

Health service use and direct outpatient costs for study participants

Of the 970 women in the ACHOIS trial with a singleton pregnancy, 474 were assigned to the Intervention Group and 496 to the Routine-Care Group. Women in the Intervention Group made 0.7 fewer antenatal clinic visits $(\mathrm{p}=$ $0.0002)$, but 2.5 more specialist medical clinic visits ( $\mathrm{p}<$ $0.0001), 1.56$ more dietician visits $(\mathrm{p}<0.0001), 1.79$ more diabetes educator visits $(\mathrm{p}<0.0001)$, and received insulin therapy more often (adjusted RR 6.18, 95\%CI 3.69 to $10.35, \mathrm{p}<0.0001)$ than women in the RoutineCare Group (Table 2). Thus, across these five types of hospital antenatal outpatient services, the mean direct costs were $\$ 337$ greater for women in the Intervention Group compared with women in the Routine-Care Group (Table 3).

\section{Primary clinical outcomes}

Singleton infants in the Intervention Group were less likely to experience any serious perinatal outcome than infants in the Routine-Care Group (adjusted RR 0.33, $95 \% \mathrm{CI} 0.14$ to $0.76, \mathrm{p}=0.01$ ), but were more likely to be admitted to a neonatal nursery (adjusted RR 1.15, 95\%CI 1.04 to $1.26, \mathrm{p}=0.004$ ) (Table 4). Women with a singleton pregnancy in the Intervention Group were more likely to have an induction of labour (adjusted RR $=1.34$, $95 \%$ CI 1.13 to $1.60, \mathrm{p}=0.001$ ), but were no more likely to have a caesarean delivery (Table 4 ).

\section{Inpatient costs}

The inpatient costing sample consisted of 195 participating women with a singleton pregnancy from the WCH. Compared to the remainder of the women with a singleton pregnancy in the ACHOIS trial, this sample was similar in maternal age and body mass index, but more likely to be nulliparous $(\mathrm{p}=0.01)$, Caucasian $(\mathrm{p}=0.01)$, and to be of lower socioeconomic status $(p=0.03)$. At trial entry these women had a higher OGTT fasting result $(\mathrm{p}=$ $0.005)$, but similar two hour OGTT result $(\mathrm{p}=0.52)$, and had a five-day greater gestational age $(\mathrm{p}<0.0001)$ compared to the remainder of women in the trial. The mean days of initial postnatal hospital stay for both these singleton women and their infants at the $\mathrm{WCH}$, with adjustment for year of admission, were not significantly different from that for women in the other participating hospitals. At the $\mathrm{WCH}$, the direct costs for inpatient services for women and infants (singleton) were $\$ 202$ greater in the Intervention Group compared with women and infants in the Routine-Care Group, not a statistically significant difference $(\mathrm{p}=0.84)$.

\section{Charges to participating women and their families}

Of the 108 women with a singleton pregnancy providing data from the questionnaire survey, the mean adjusted charge from randomisation until birth, for women in the 
Table 2: Health services use after enrolment by women with a singleton gestation and their infants

\begin{tabular}{|c|c|c|c|c|}
\hline & Intervention group & Routine-care group & Adjusted treatment effect ${ }^{\#}(95 \% \mathrm{Cl})$ & $\begin{array}{c}\text { Adjusted } \\
\text { p-value }\end{array}$ \\
\hline & $n=474$ & $n=496$ & & \\
\hline \multicolumn{5}{|c|}{ Antenatal services after enrolment } \\
\hline Antenatal inpatient admission & $135(28.5)$ & $133(26.8)$ & $1.11(0.91-1.36)$ & 0.31 \\
\hline Antenatal clinic visits* & $4.4(I-7)$ & $5.2(3-7)$ & $-0.70(-1.06,-0.33)$ & 0.0002 \\
\hline Specialist clinic visits* & $4.0(1-7)$ & $\mathrm{I} .3(0-2)$ & $2.50(2.13,2.87)$ & $<0.0001$ \\
\hline Dietician visits* & $\mathrm{I} .7(\mathrm{I}-2)$ & $0.2(0-0)$ & $1.56(1.39,1.72)$ & $<0.0001$ \\
\hline Diabetes educator visits* & $2.0(I-2)$ & $0.2(0-0)$ & $1.79(1.59,1.98)$ & $<0.0001$ \\
\hline Insulin therapy* & $96(20.3)$ & $16(3.2)$ & $6.18(3.69-10.35)$ & $<0.0001$ \\
\hline \multicolumn{5}{|l|}{ Birthing services } \\
\hline Induction of labour & $183(38.6)$ & $148(29.8)$ & $1.34(1.13-1.60)$ & 0.001 \\
\hline Caesarean birth & $142(30.0)$ & $153(30.8)$ & $0.97(0.80-1.17)$ & 0.76 \\
\hline \multicolumn{5}{|l|}{ Neonatal services } \\
\hline Paediatrician present at delivery & $211(44.5)$ & $225(45.4)$ & $1.02(0.89-1.17)$ & 0.72 \\
\hline Admission to neonatal nursery & $330(69.6)$ & $294(59.3)$ & $1.15(1.04-1.26)$ & 0.004 \\
\hline Hours of phototherapy§ & $45(24-72)$ & $36(24-51)$ & & 0.27 \\
\hline
\end{tabular}

Dichotomous outcomes reported as number (\%) of women/babies with relative risk and $95 \%$ confidence intervals $(\mathrm{Cl})$ as treatment effect; *continuous outcomes reported as mean (interquartile range) with mean difference $95 \% \mathrm{Cls}$ as treatment effect.

$\S$ Hours of phototherapy is median (interquartile range).

\#Adjusted for maternal age, race or ethnic group and parity.

Intervention Group and their families, was $\$ 367$ compared with $\$ 302$ for the Routine-Care Group ( $\mathrm{p}=0.34$ ) (Table 5).

\section{Costs and consequences of treatment for mild GDM}

Over the course of the ACHOIS trial, for every 100 women with a singleton pregnancy and a positive OGTT who were offered treatment of mild gestational diabetes mellitus in addition to standard obstetric care (Table 6),

- \$53,985 additional direct costs were incurred at the obstetric hospital,

- \$6,521 additional charges were incurred by the women and their families,
- 9.7 additional women experienced an induction of labour, and

- 8.6 more babies were admitted to a neonatal nursery, yet

- 2.2 fewer babies experienced any serious perinatal complication (includes perinatal death, shoulder dystocia, bone fracture, and nerve palsy), and

- 1.0 fewer babies experienced a perinatal death.

\section{Incremental cost-effectiveness ratio}

Although a cost-consequences analysis was planned, the actual trial results enabled the estimation of a credible cost-effectiveness ratio in terms of the incremental cost for a reduction in any serious perinatal complication. The

Table 3: Adjusted mean direct hospital outpatient costs for women with a singleton gestation

\begin{tabular}{crrr}
\hline & $\begin{array}{r}\text { Intervention group } \\
(\mathbf{n}=\mathbf{4 7 4})\end{array}$ & $\begin{array}{r}\text { Routine-care group } \\
(\mathbf{n}=\mathbf{4 9 6})\end{array}$ & Difference in adjusted mean cost \\
& Adjusted mean cost & Adjusted mean cost & $-\$ 20$ \\
\hline Antenatal clinic visits & $\$ 125$ & $\$ 145$ & $\$ 174$ \\
Specialist clinic visits & $\$ 327$ & $\$ 153$ & $\$ 76$ \\
Dietician visits & $\$ 87$ & $\$ 11$ & $\$ 57$ \\
Diabetes educator & $\$ 67$ & $\$ 10$ & $\$ 51$ \\
Insulin therapy & $\$ 69$ & $\$ 337$ & $\$ 337$ \\
Total & $\$ 674$ &
\end{tabular}

Unit costs from Table I. Occasions of service from main ACHOIS data base.

Adjusted for maternal age, race or ethnic group, and parity.

Rounded to whole dollars 
Table 4: Primary clinical outcomes among women with a singleton gestation and their infants

\begin{tabular}{|c|c|c|c|c|}
\hline & Intervention group & Routine-care group & Adjusted RR (95\% Cl) & Adjusted p-value \\
\hline Infants & $n=474$ & $n=496$ & & \\
\hline Any serious perinatal complication & $7(1.5)$ & $23(4.6)$ & $0.33(0.14-0.76)$ & 0.01 \\
\hline Death & $0(0.0)$ & $5(1.0)$ & & 0.07 \\
\hline Shoulder dystocia & $7(1.5)$ & $16(3.2)$ & $0.46(0.19-1.12)$ & 0.09 \\
\hline Bone fracture & $0(0.0)$ & I $(0.2)$ & & 0.38 \\
\hline Nerve palsy & $0(0.0)$ & $3(0.6)$ & & 0.11 \\
\hline Admission to neonatal nursery & $330(69.6)$ & $294(59.3)$ & $1.15(1.04-1.26)$ & 0.004 \\
\hline Jaundice requiring phototherapy & $44(9.3)$ & $42(8.5)$ & $1.06(0.7 \mathrm{I}-1.59)$ & 0.76 \\
\hline Women & $n=474$ & $n=496$ & & \\
\hline Induction of labour & $183(38.6)$ & $148(29.8)$ & $1.34(1.13-1.60)$ & 0.001 \\
\hline Caesarean delivery & $142(30.0)$ & $153(30.8)$ & $0.97(0.80-1.17)$ & 0.76 \\
\hline
\end{tabular}

Values are number $(\%)$ of babies or women

Adjusted for maternal age, race or ethnic group, and parity

incremental cost per additional serious perinatal complication prevented (defined prospectively as one or more of the following: death, shoulder dystocia, bone fracture, and nerve palsy) was therefore $\$ 27,503(=(\$ 53,985+$ $\$ 6,521) / 2.2)$. The incremental cost per perinatal death prevented was $\$ 60,506(=(\$ 53,985+\$ 6,521) / 1.0)$. Based on Australian life tables at the midpoint of the trial [14], and a discount rate of $5 \%$, the incremental cost per lifeyear saved was $\$ 2,988(=(\$ 53,985+\$ 6,521) / 20.25)$.

\section{Discussion}

The ACHOIS trial [2] has demonstrated the clinical effectiveness of active treatment of women with mild gestational diabetes and this paper reports the costeffectiveness of such treatment. Although a cost-consequence analysis was planned, the actual trial data allowed a credible cost-effectiveness ratio to be estimated, demonstrating that the form of an economic analysis often cannot be settled until data on effectiveness and cost are actually available [15]. The ACHOIS trial also demonstrated that the health-related quality of life of the inter- vention mothers was better, both during the antenatal period and three months after birth [2].

Our economic analysis used primary clinical outcome information and use of hospital services for all 970 women with a singleton pregnancy recruited to the ACHOIS trial. For assessing inpatient resource use, the relative differences in costs incurred by women and infants at the hospital contributing the largest number of women ( $n=195)$ were assumed to be representative of those across the whole study. This representation was used because the trial began before computerised inpatient cost information systems became routinely available in the participating hospitals.

The size of this study was limited by the sample size of 1000 women in the ACHOIS trial and the small sample of women able to be assessed for charges to families. The power of the study to show differences was therefore low. Even with these limitations however, these data are of importance as there have been no previous reports of costs

Table 5: Mean charges* to women and their family from randomisation to birth.

\begin{tabular}{crr}
\hline & Intervention group (n= 52) & Routine-care group (n= 56) \\
Paid child care & Adjusted mean & Adjusted mean \\
Travel (to \& from appts.) & $\$ 24$ & $\$ 13$ \\
Blood glucose monitoring equipment \& consumables & $\$ 46$ & $\$ 42$ \\
Food substitution: additional expenditure & $\$ 53$ & $\$ 17$ \\
Subtotal & $\$ 20$ & $\$ 10$ \\
Mother time off paid work & $\$ 83$ & $\$ 145$ \\
Partner time off work & $\$ 141$ & $\$ 75$ \\
Total & $\$ 367$ & $\$ 302$ \\
\hline
\end{tabular}

* In 2002 Australian dollars, pecuniary and non-pecuniary costs. Wages calculated at the rate of Average Weekly Earnings (ABS).

Adjusted for maternal age, race or ethnic group, and parity.

Rounded to whole dollars.

Costs did not differ between groups 
Table 6: Summary of direct costs post-randomisation and consequences of management of mild gestational diabetes mellitus expressed per 100 women with a singleton gestation

\begin{tabular}{|c|c|c|c|}
\hline & Intervention group $(n=100)$ & Routine-care group $(n=100)$ & Difference $(n=100)$ \\
\hline \multicolumn{4}{|l|}{ Direct costs post-randomisation- } \\
\hline \multicolumn{4}{|l|}{ Antenatal ambulatory services * } \\
\hline Antenatal clinic & $\$ 12,479$ & $\$ 14,483$ & $\$-2,004$ \\
\hline Specialist clinic & $\$ 32,657$ & $\$ 15,290$ & $\$ 17,367$ \\
\hline Dietician & $\$ 8,716$ & $\$ 1,127$ & $\$ 7,589$ \\
\hline Diabetes educator & $\$ 6,692$ & $\$ 982$ & $\$ 5,710$ \\
\hline Insulin therapy & $\$ 6,888$ & $\$ 1,800$ & $\$ 5,088$ \\
\hline Subtotal & $\$ 67,432$ & $\$ 33,681$ & $\$ 33,751$ \\
\hline \multicolumn{4}{|l|}{ Inpatient services } \\
\hline Hospital costs $\dagger$ & $\$ 545,125$ & $\$ 524,891$ & $\$ 20,234$ \\
\hline Total direct health service costs & $\$ 6 / 2,557$ & $\$ 558,572$ & $\$ 53,985$ \\
\hline Patient/family costs $\ddagger$ & $\$ 36,749$ & $\$ 30,229$ & $\$ 6,521$ \\
\hline \multicolumn{4}{|l|}{ Consequences $\S$} \\
\hline \multicolumn{4}{|l|}{ Infants } \\
\hline Any serious perinatal complication $\mathbb{T}$ & I.I & 3.2 & -2.2 \\
\hline Admission to neonatal nursery & 68.1 & 59.5 & 8.6 \\
\hline \multicolumn{4}{|l|}{ Women } \\
\hline Induction of labour & 38.0 & 28.3 & 9.7 \\
\hline
\end{tabular}

*Antenatal ambulatory services post-randomisation based on ACHOIS trial data for 970 women (474 in Intervention group and 496 in Routine care group respectively).

† Hospital costs for all mothers and babies based on WCH patient costing system (obtained from data for 195 inpatient women with a singleton pregnancy at the $\mathrm{WCH}$ ).

$\ddagger$ Patient/family costs obtained by survey of a patient subsample of 108 South Australian study participants.

$\S$ Based on ACHOIS trial data for 474 and 496 singleton women (respectively) (see Table 4).

Therious perinatal complications were defined prospectively as one or more of the following: death, shoulder dystocia, bone fracture, and nerve palsy.

Adjusted for maternal age, race or ethnic group, and parity.

of treatment options for women with gestational diabetes from randomised trials.

It is likely that the general public in high-income countries such as Australia would find the reduction in perinatal mortality sufficient to justify the additional costs of $\$ 60,506$, whether or not society places a larger value on a baby's life than on that of other members of the general public. Even incremented to current year costs, the figures would remain highly favourable. Indeed, at a value of $\$ 2,988$, the incremental cost per life-year gained is highly favourable. By way of comparison, George et al. [16] found that historically the Australian Pharmaceutical Benefits Advisory Committee was unlikely to recommend a drug for government subsidy if the additional cost per lifeyear gained exceeded $\$ 86,154$ and was unlikely to reject a drug for which the additional cost per life-year gained was less than $\$ 47,612$ (2002 Australian dollar values).

The results suggest that being diagnosed with mild gestational diabetes mellitus and receiving the recommended care is not associated with any significant increase in the direct inpatient costs or postnatal length of stay, but is associated with an increase in the women's antenatal out- patient costs. This includes costs due to an increase in the number of visits to a physician, dietician and diabetes educator. The economic evaluation has been confined to in-trial costs and consequences. No modelling has yet been attempted to account for either costs or consequences beyond the end of the trial.

\section{Conclusion}

Treatment of women with gestational diabetes by dietary advice, blood glucose monitoring and insulin therapy as needed resulted in a reduction in serious perinatal complications, although more women experienced an induction of labour, and more of their infants were admitted to the neonatal nursery [2]. Treatment of women with a singleton pregnancy who have gestational diabetes in this way resulted in increased direct costs of outpatient, obstetric hospital services and direct out of pocket charges for women and families compared with routine pregnancy care. However, taking a perspective over the whole lifespan, the incremental cost per extra life-year gained is highly favourable. It is likely that the general public in high-income countries such as Australia would find the reductions in perinatal mortality and in serious perinatal 
complications sufficient to justify the additional health service and personal monetary costs.

\section{Competing interests}

The author(s) declare that they have no competing interests.

\section{Authors' contributions}

All authors contributed to the study design, interpretation of the data and preparation of the drafts of the manuscript. In addition CC coordinated the trial and the collection of data. KW performed the data analyses. All authors read and approved the final manuscript.

\section{Acknowledgements}

We are indebted to the women and their children who participated in this study; to SJ Alton, A Deussen, I Flight, E Griffith, J Lumley, A Thomas and L Watson for data collection, management and monitoring; to S Brown, $\mathrm{K}$ Bruggemann and $P$ Moore for providing the in-patient cost data; and to $M$ Ewens for administrative assistance with the manuscript.

The following persons and institutions participated in the ACHOIS Trial Group

Blacktown District Hospital, New South Wales: D. Chipps, R. Myszka, S. Hendon, M. McLean, H. Merker, J. Bradford; Bradford Royal Infirmary Maternity Unit, United Kingdom: D. Tuffnell, J. West; Caboolture Hospital, Queensland: M. Ratnapala, R. Hinton, D. Woodford, D. Cave, C. Armstrong, A. Vacca, P. Joubert, S. Mego, V. Heazelwood; Campbelltown Hospital, Sydney, New South Wales: H. Grunstein, S. Fleming, B. Marney; Flinders Medical Centre, Adelaide, South Australia: K. Harris, J. Ebert, R. Bryce, S. Judd, M. Keirse, C. Verco; General Infirmary, Leeds, United Kingdom: E. Ferriman, G. Mason, C. LidelleJohnson, J. Pearce; Hammersmith Hospital, London: M. de Swiet, A. McCarthy; Hervey Bay Hospital, Queensland: A. Lindberg, D. Ludwig, K. Wickremachandran; Lyell McEwin Hospital, Adelaide, South Australia: G. Dekker, P. Duggan, I. Hocking, W. Jeffries, S. Kennedy-Andrews, N. Kretschmer, H. Millar, J. Mowbray; Modbury Hospital, Adelaide, South Australia: C. Archer, C. Hughes, G. Matthews, M. Morton, N. Price, L. Purins, N. Tamlin, J. Sieben; Nambour General Hospital, Queensland: C. Cocks, M. Gregora, S. Hamwood, G. Pinn, C. Rutherford, C. Sheehan, T. Stubbs, V. Smith-Orr; Northern General Hospital, Sheffield, United Kingdom: S. Rutter, C. Bruce, R. Fraser; Queen Elizabeth Hospital, Adelaide, South Australia: B. Pridmore (deceased), W. Hague, P. Phillips, M. Sladek, S. Torr; Royal North Shore Hospital, Sydney: G. Burton, R. Hitchman, I. Kelso, A. McElduff, J. Morris; St. George Hospital, Sydney: C. Homer, G. Davis; Toowoomba Base Hospital, Queensland: P. Bridger, Y. Chadha, D. Gibson, M. Ratnapala; Townsville Hospital, Queensland: D. Watson, A. Rane, A. Robinson, J. Whitehall, S. Dunstone, R. Chadwick, A. Dederer, A. Lawrence; Women's and Children's Hospital, South Australia: C. Crowther, R. Burnet, A. McPhee, J. Robinson, A. Thomas, S. Alton, J. Hayton, J. Paynter, A. Deussen, J. Avery, S. Agett, D. Morris, B. Peat, C. Wilkinson, V. Coppinger, J. Dodd.

\section{References}

I. Drummond MF, Sculpher MJ, Torrance GW, O'Brien BJ, Stoddart GL: Methods for the economic evaluation of health care $3 r d$ edition. Oxford University Press; 2005

2. Crowther CA, Hiller JE, Moss JR, McPhee AJ, Jeffries WS, Robinson JS: Effect of treatment of gestational diabetes mellitus on pregnancy outcomes. NEIM 2005, 2:2477-86.

3. Diabetes mellitus: report of a WHO study group. World Health Organ Tech Rep Ser 1985, 727:1-1 I3.
4. Greenhalgh T: Papers that tell you what things cost (economic analyses). British Medical Journal 1997, 3 I 5:596-9.

5. Coast J: Is economic evaluation in touch with society's health values? British Medical Journal 2004, 329:1233-6.

6. Commonwealth Department of Health and Ageing: Manual of resource items and their associated costs for use in major submissions to the Pharmaceutical Benefits Advisory Committee involving economic analyses Canberra: AusInfo; 2002.

7. Commonwealth Department of Health and Ageing: Medicare Benefits Schedule Book Canberra: Information Services. I November 200 I (including I May 2002 update)

8. Department of Health and Ageing: Schedule of Pharmaceutical Benefits for approved Pharmacists and Medical Practitioners Canberra: Commonwealth Copyright Administration; 200I.

9. Department of Veterans' Affairs for Allied Health Services Fees [http://www.dva.gov.au/health/provider/provider.htm]

10. Commonwealth Department of Health and Ageing: National Hospital Cost Data Collection. Cost report round 7 (2002-2003) Canberra; 2004.

1I. Australian Bureau of Statistics: Consumer Price Index. (Cat no. 640 I.0) Canberra: ABS; 2003

12. Organisation for Economic Co-operation and Development [http://www.oecd.org/std/ppp]

13. Barber JA, Thompson SG: Analysis and interpretation of cost data in randomised controlled trials: review of published studies. BMJ 1998, 3 I7(7 I67): I | 95- 1200.

14. Australian Bureau of Statistics: Deaths, Australia 1999. (Cat No. 3302.0) Canberra: ABS; 2000.

15. Donaldson C, Hundley V, Mclntosh E: Using economics alongside clinical trials: Why we cannot choose the evaluation technique in advance. Health Economics 1996, 5:267-269.

16. George B, Harris A, Mitchell A: Cost-effectiveness analysis and the consistency of decision making: Evidence from pharmaceutical reimbursement in Australia 199/-96. Pharmacoeconomics 200I, 19:1 103-9.

\section{Pre-publication history}

The pre-publication history for this paper can be accessed here:

http://www.biomedcentral.com/1471-2393/7/27/prepub

Publish with BioMed Central and every scientist can read your work free of charge

"BioMed Central will be the most significant development for disseminating the results of biomedical research in our lifetime. " Sir Paul Nurse, Cancer Research UK

Your research papers will be:

- available free of charge to the entire biomedical community

- peer reviewed and published immediately upon acceptance

- cited in PubMed and archived on PubMed Central

- yours - you keep the copyright

Submit your manuscript here:

http://www.biomedcentral.com/info/publishing_adv.asp
BioMedcentral 